\title{
Femtosecond-Laser-Induced Nanostructure and High Ablation Rate Observed on Nitrided Alloy Steel
}

\author{
Naoki YASUMARU ${ }^{* 1}$, Eisuke SENTOKU ${ }^{* 1}$, Masakazu HAGA ${ }^{* 1}$ and Junsuke KIUCHI ${ }^{* 2}$ \\ ${ }^{* 1}$ Fukui National College of Technology, Geshi-cho, Sabae, Fukui 916-8507, Japan \\ E-mail: yasuma@fukui-nct.ac.jp \\ ${ }^{* 2}$ Eyetec Co., Ltd., 2-6-8 Kaminaka-cho, Sabae, Fukui 916-0016, Japan
}

\begin{abstract}
Femtosecond (fs)-laser-induced nanostructuring of mainly nitrided die steel $(1.5 \mathrm{C}-12 \mathrm{Cr})$ was experimentally investigated using die steel that was heat-treated and then plasma nitrided. Nitrided stainless steel $(18 \mathrm{Cr}-8 \mathrm{Ni})$ and low-carbon steel $(0.1 \mathrm{C})$ were also used to examine the influence of the alloy composition. Periodic nanostructures were formed in the direction perpendicular to the laser polarization and the mean spacing $D$ increased with the fluence $F$. The minimum $D$ values for alloy steels containing Cr observed at $F$ around the ablation threshold were 250-285 nm, which corresponds to a size of about $\lambda / 3$. In contrast, the minimum $D$ for low-carbon steel was larger at 20 to $30 \%$ more than those of the alloy steels. In addition, the ablation rate was obtained by analysis of the cross-sectional profiles of craters ablated by superimposed fs-laser pulses. Although the ablation rates for the non-nitrided steels increased linearly with $F$ on a logarithmic scale, it should be noted that the ablation rate for nitrided die or stainless steels increased faster than linearly with $F$.

DOI: $10.2961 /$ jlmn.2015.01.0007
\end{abstract}

Keywords: femtosecond-laser ablation, nanostructure, alloy steel, nitriding, tribology

\section{Introduction}

Surface hardening of alloy steels by plasma-nitriding is well known to improve its wear resistance, fatigue resistance and antiseizure properties [1,2]. The nitrided layer formed on steel tends to receive heat influence, so that further improvement of its tribological properties may be expected when the nitrided surface is non-thermally nanostructured and covered with a solid or liquid lubricant. We have previously demonstrated that the frictional performance of diamond-like carbon (DLC) film is significantly improved by nanostructuring the surface with femtosecond (fs)-laser pulses and sputter-coating with $\mathrm{MoS}_{2}$ [3]. The formation of fs-laser-induced nanostructure can be attributed to the excitation of surface plasmon polaritons to induce the periodic enhancement of local fields in the surface layer $[4,5]$. It is expected that this technique of nanostructuring can be used for a variety of solid-state materials.

Our previous studies [6,7] have demonstrated that periodic nanostructures are formed on nitrided stainless steel, and the mean spacing $D$ for the nanostructure has a minimum of $250 \mathrm{~nm}$ around the ablation threshold, which corresponds to a size of about $\lambda / 3$, and $D$ increases with the fluence $F$. Furthermore, $D$ was 10 to $20 \%$ smaller for the nitrided surface than that for the untreated surface and a characteristic swelling phenomenon of an irradiated area on the nitrided layer was observed.

In this paper, fs-laser-induced nanostructuring of mainly nitrided die steel $(1.5 \mathrm{C}-12 \mathrm{Cr})$, which is utilized for the metal molds and machine parts, was experimentally investigated. The die steel was heat-treated (quenched and tempered at low temperature) and then plasma nitrided. Although the formation of periodic nanostructure on the nitrided die steel is effective for improving the durability of metallic molds, or the mold-release characteristics, the nanostructure must be processed as much as possible at low temperature.

The morphological changes of the irradiated specimens were analyzed using scanning electron microscopy (SEM), and the nanostructure features formed on the nitrided die steel were compared with those for heat-treated die steel. Further, to elucidate the influence of alloy composition, fslaser-induced nanostructuring was also examined for nitrided stainless steel $(18 \mathrm{Cr}-8 \mathrm{Ni})$ and untreated low-carbon steel (0.1C). The stainless steel contained a similar amount of chromium as the die steel, although the carbon content was lower, and the low-carbon steel did not contain chromium. Therefore, it was considered that the influence of the alloy elements could be clarified by comparison of these three steels.

In addition, the ablation rates were measured by scanning probe microscopy (SPM) analysis of the crosssectional profiles of craters ablated by superimposed fslaser pulses to investigate the ablation phenomenon of the nitrided steel surface, and the obtained rates were plotted against $F$ on a logarithmic scale. The characteristics of the nitrided layer after the fs-laser ablation were determined by a comparison of nitrided and non-nitrided steels in terms of the ablation rate dependence on the fluence.

\section{Experimental procedure}

Commercially available, surface-polished 3-mm-thick die steel $(1.5 \mathrm{C}-12 \mathrm{Cr})$ plates were utilized. The die steel was heat-treated by quenching in oil and then tempering at low temperature. Some of these plates were subjected to plasma-nitriding by DC glow discharge plasma in an $\mathrm{N}_{2}-\mathrm{H}_{2}$ mixed gas at approximately $723 \mathrm{~K}$. The surface of plasmanitrided specimen was progressively removed with emery paper down to \#1200 and then polished with diamond paste $(3 \mu \mathrm{m})$. The heat-treated die steel had a Vickers hardness 
(HV) of 680 , whereas the nitrided die steel had a much higher HV of 1050.

Surface-polished type 304 austenitic stainless steel $(18 \mathrm{Cr}-8 \mathrm{Ni})$ and low-carbon steel $(0.1 \mathrm{C})$ plates were also utilized to examine the influence of the alloy composition. Samples of the stainless steels were plasma-nitrided at approximately $773 \mathrm{~K}$. The untreated stainless steel and lowcarbon steel had HVs of 300 and 180, respectively, whereas that of the nitrided stainless steel was much higher at 1300 .

These specimens were irradiated in air with linearly polarized, $800 \mathrm{~nm}, 180$ fs laser pulses from a Ti:sapphire chirped-pulse amplification system operated at a repetition frequency of $1 \mathrm{kHz}$. The fs laser pulses with a pulse energy of 150-600 $\mathrm{J}$ were focused on the target surface using a parabolic mirror with a $200 \mathrm{~cm}$ focal length. The laser fluence at the target was estimated to be $F=0.06-0.24 \mathrm{~J} / \mathrm{cm}^{2}$. The target plate was mounted on a precision $\mathrm{X}-\mathrm{Y}$ stage. The stage was fixed and a number of laser pulses of $N=$ 10-500 was superimposed on the target plate at $1 \mathrm{kHz}$. The ablated spot size on the target was typically about 250 $\mu \mathrm{m}$.

Optical microscopy, field-emission SEM (Hitachi Ltd., S-4100) and SPM (Seiko Instruments Inc., Nanopics 2100) were used to examine the morphological changes of the irradiated specimens. The $D$ value for the periodic nanostructure formed on the specimen surface was determined from the peak position of the power spectrum obtained by Fourier transform of SEM images of the nanostructure using image processing software (Image Metrology A/S, SPIP ${ }^{\mathrm{TM}}$ ) [7].

\section{Results and discussion}

\subsection{Nanostructure formation}

(a)

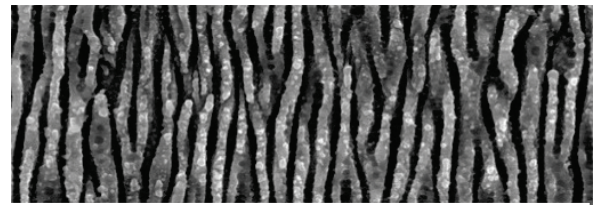

(b)

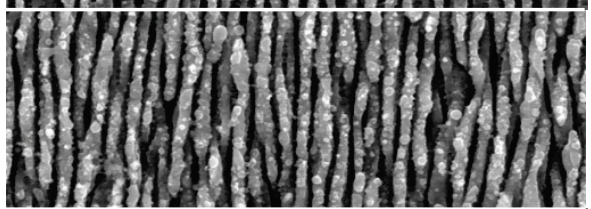

(c)

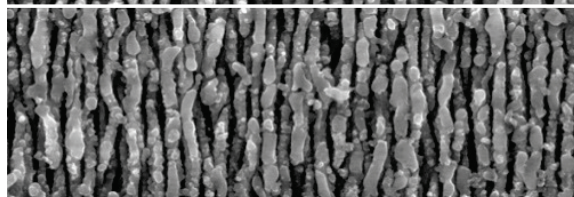

(d)

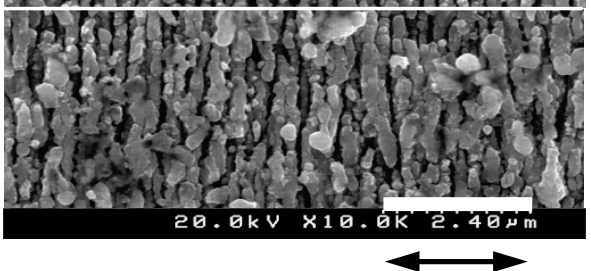

Fig. 1 SEM images of nitrided die steel surfaces ablated by superimposed fs-laser pulses of $N=$ (a) 50, (b) 100, (c) 200 and (d) 500 at a fluence of $0.08 \mathrm{~J} / \mathrm{cm}^{2}$. The arrow indicates the polarization direction of the fs-laser pulses.
Figure 1 shows SEM images near the center of the nitrided die steel plate surfaces irradiated with fs-laser pulses of $N=50,100,200$, and 500 at $F=0.08 \mathrm{~J} / \mathrm{cm}^{2}$, just above the ablation threshold. The periodic nanostructures formed are almost oriented in the direction perpendicular to the laser polarization. At $N=50$, a nanostructure with $D \sim 310$ $\mathrm{nm}$ is uniformly generated on the focal spot. The period decreases to $D \sim 285 \mathrm{~nm}$ at $N=200$.

Figure 2 shows SEM images of the nitrided die steel surfaces ablated at a higher $F$ of $0.2 \mathrm{~J} / \mathrm{cm}^{2}$ for different $N$ values. At this fluence, periodic nanostructures with a large $D$ of $660 \mathrm{~nm}$ were formed at $N=10$, and decreased to $560 \mathrm{~nm}$ at $N=50$. $D$ gradually increased to $620 \mathrm{~nm}$ with an increase in the number of laser pulses to $N=500$.

(a)

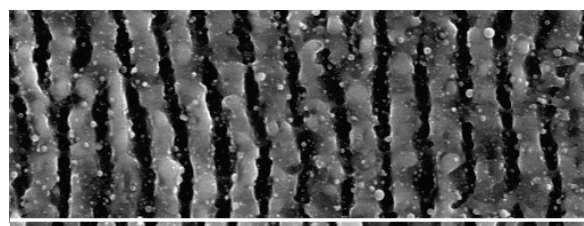

(b)

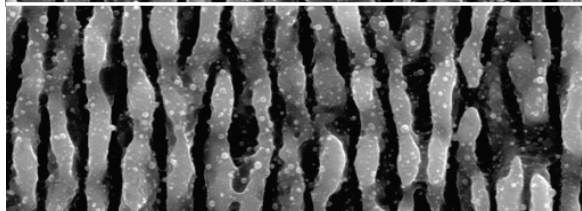

(c)

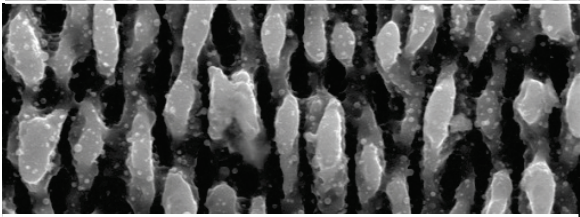

(d)

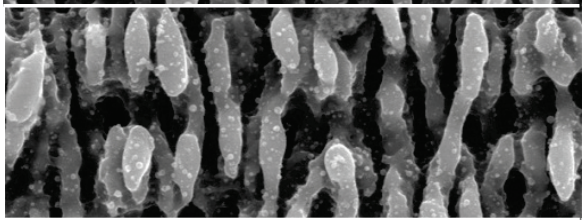

(e)

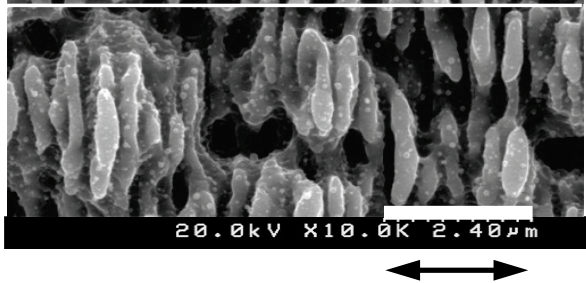

Fig. 2 SEM images of nitrided die steel surfaces ablated by superimposed fs-laser pulses of $N=$ (a) 10, (b) 50, (c) 100, (d) 200 and (e) 500 at a fluence of $0.2 \mathrm{~J} / \mathrm{cm}^{2}$.

Figure 3 presents the variation in $D$ determined from the Fourier power spectra for nanostructures formed on heat-treated and nitrided die steel, as a function of $N$ at different incident fluences of $F=0.08,0.12,0.16$, and 0.2 $\mathrm{J} / \mathrm{cm}^{2}$. As the fluence is decreased, the number of pulses for which periodic nanostructures are uniformly formed increases from 10 to approximately 50, although $D$ for the resulting nanostructure becomes smaller. The minimum $D$ was obtained at $0.08 \mathrm{~J} / \mathrm{cm}^{2}$ near the ablation threshold and was $285 \mathrm{~nm}$ for the nitrided die steel and $265 \mathrm{~nm}$ for the heat-treated die steel at $N=200$, where these values correspond to approximately $1 / 3$ of the laser wavelength $(\lambda / 3)$, as for the case of nitrided stainless steel $[6,7]$. 
When $N$ was small, $D$ for the nitrided die steel increased, i.e., at $N=10$ and at a higher fluence $>0.12 \mathrm{~J} / \mathrm{cm}^{2}$, $D$ was $600-670 \mathrm{~nm}$, which is equivalent to $4 / 5 \lambda$. However, $D$ decreased with increasing of $N$ and almost plateaued. This phenomenon was also observed for stainless steel.

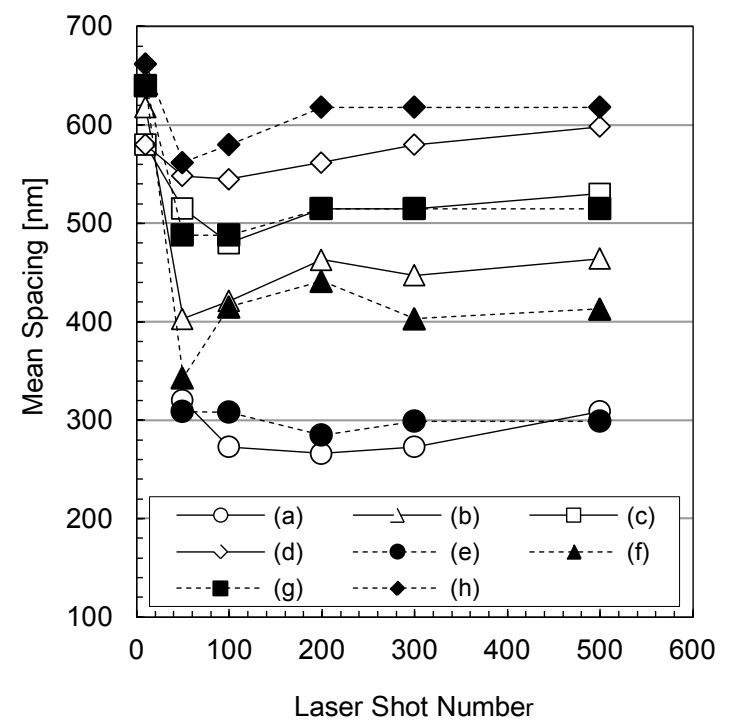

Fig. 3 Mean spacing $(D)$ of periodic nanostructures formed on $(\mathrm{a}-\mathrm{d})$ heat-treated die steel and $(\mathrm{e}-\mathrm{h})$ nitrided die steel, measured as a function of $N$ at $F=(\mathrm{a}, \mathrm{e}) 0.08,(\mathrm{~b}, \mathrm{f}) 0.12,(\mathrm{c}, \mathrm{g}) 0.16$ and $(\mathrm{d}, \mathrm{h})$ $0.2 \mathrm{~J} / \mathrm{cm}^{2}$.

Although $D$ for the fs-laser-induced periodic structures formed on metals are larger than $\lambda / 2$ in many cases [8-10], it was reported that $D$ was $300-500 \mathrm{~nm}$ for austenitic hightemperature steel [11], and nanostructures with $D=310 \mathrm{~nm}$ were formed on stainless steel at a low pulse energy [12]. In addition, Hou et al. reported that short periodic ripples with $D=310-270 \mathrm{~nm}$ were formed at $F=0.07-0.13 \mathrm{~J} / \mathrm{cm}^{2}$ [13]. We have previously reported [6,7] almost the same minimum $D$ for stainless steel; however, $D$ increases gradually with an increase of $F$ from 0.08 to $0.16 \mathrm{~J} / \mathrm{cm}^{2}$ at $\mathrm{N}>$ 100 .

We have also reported that the nanostructures on the nitrided stainless steel had smaller $D$ than that for untreated stainless steel under the same experimental conditions. The minimum $D$ for nanostructures formed on $\mathrm{CrN}$ is reported to be very small at about $110 \mathrm{~nm}$ [14]; therefore, it may be considered that precipitation of $\mathrm{CrN}$ nitrides in the nitrided layer causes a reduction of $D$.

In contrast, $D$ for the nanostructure formed on the nitrided die steel seldom differs from that formed on the heattreated die steel, as shown in Fig. 3. $\mathrm{Cr}_{7} \mathrm{C}_{3}$ carbides are already precipitated in the heat-treated die steel; therefore, it is considered that $\mathrm{Cr}_{7} \mathrm{C}_{3}$ has the same effect as $\mathrm{CrN}$ on $D$, so that there is no reduction in $D$ due to the nitriding dose.

Figure 4 shows the minimum values of $D$ observed at $F$ around the ablation threshold for heat-treated die steel, nitrided die steel, untreated stainless steel, nitrided stainless steel, and low-carbon steel. The minimum $D$ values for these $\mathrm{Cr}$ alloy steels were $250-285 \mathrm{~nm}$, which corresponds to a size of about $\lambda / 3$. However, the minimum $D$ for the low-carbon steel was $330 \mathrm{~nm}$ larger (20 to $30 \%$ ) than those for the alloy steels. The other steels contain chromium; therefore, it may be considered that chromium nitride, carbide and oxide generated on the specimen surface cause a reduction in $D$.

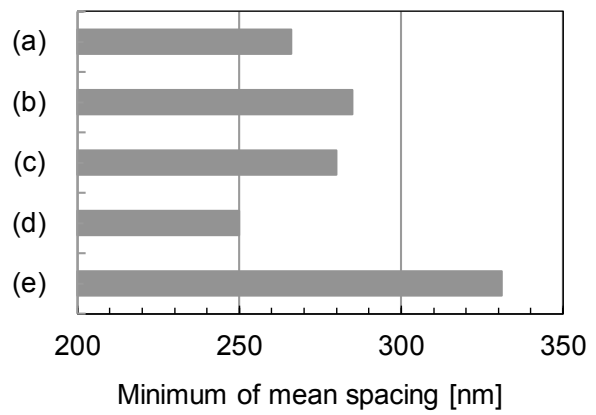

Fig. 4 Minimum $D$ values observed at $F$ around the ablation threshold for (a) heat-treated die steel, (b) nitrided die steel, (c) untreated stainless steel, (d) nitrided stainless steel and (e) lowcarbon steel

\subsection{Ablation rate}

Figure 5 shows the cross-sectional profiles of craters formed on heat-treated die steel by ablation with superimposed fs-laser pulses for $N=300$ and at various incident fluences of $F=0.08,0.10$ and $0.12 \mathrm{~J} / \mathrm{cm}^{2}$ measured using SPM. A phenomenon where an irradiated spot swells approximately $150 \mathrm{~nm}$ was evident at a low fluence of $F=$ $0.08 \mathrm{~J} / \mathrm{cm}^{2}$, although nanostructures with $D=270 \mathrm{~nm}$ were formed on the surface of the swollen area. The crater was formed at a fluence of $0.1 \mathrm{~J} / \mathrm{cm}^{2}$ or greater and the crater depth increased with $F$.

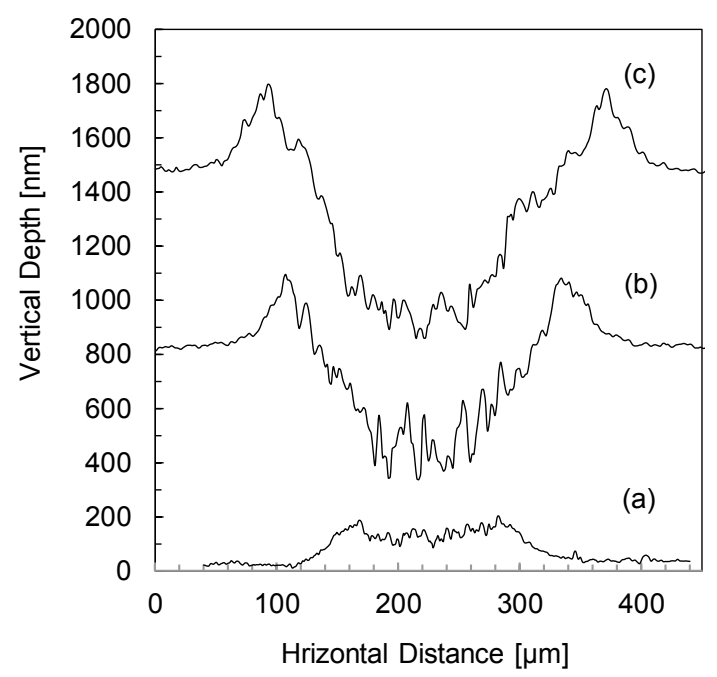

Fig. 5 Cross-sectional profiles for craters formed on heat-treated die steel by ablation with superimposed fs-laser pulses for $N=$ 300 at $F=$ (a) 0.08 , (b) 0.10 and (c) $0.12 \mathrm{~J} / \mathrm{cm}^{2}$.

This swelling was also observed on the nitrided stainless steel that was nanostructured at a low fluence of $F=$ $0.08 \mathrm{~J} / \mathrm{cm}^{2}$ [7]. The nitrided layer formed on the stainless steel has the unstable austenitic $\gamma$ phase, which was also present on the heat-treated die steel. The $\gamma$ phase may be transformed into the $\alpha$ phase by fs-laser irradiation and swelling would be induced by the expansion of the lattice volume. 
Figure 6 shows the cross-sectional profiles of craters formed on nitrided die steel by ablation with superimposed fs-laser pulses for $N=300$ and at $F=0.08,0.10$ and 0.12 $\mathrm{J} / \mathrm{cm}^{2}$. A crater was formed on an irradiated spot and the depth of the crater increased from 110 to $1600 \mathrm{~nm}$ with increasing $F$.

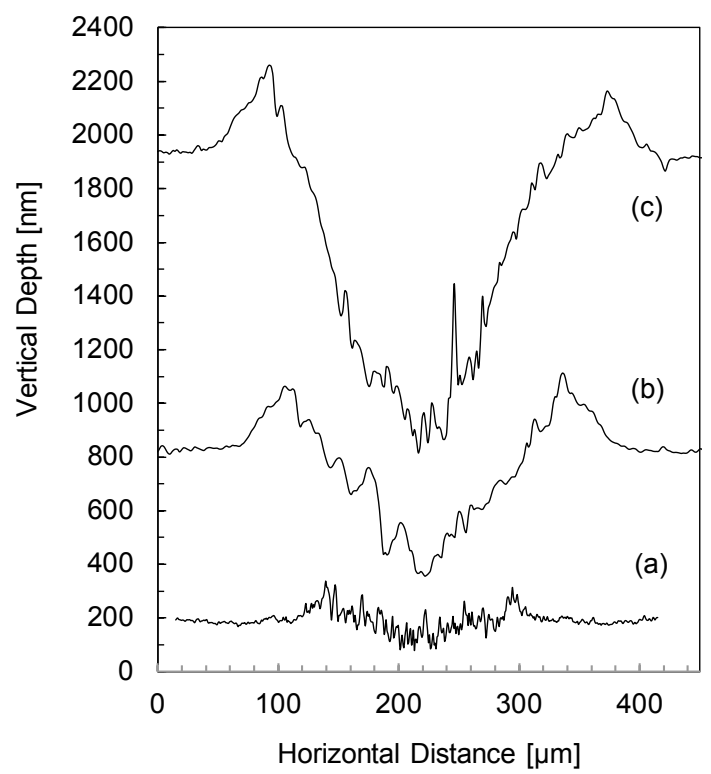

Fig. 6 Cross-sectional profiles for craters formed on nitrided die steel by ablation with superimposed fs-laser pulses for $N=300$ at $F=$ (a) 0.08 , (b) 0.10 and (c) $0.12 \mathrm{~J} / \mathrm{cm}^{2}$.

Swelling was no longer observed on the nitrided die steel that was nanostructured at a low fluence of $F=0.08$ $\mathrm{J} / \mathrm{cm}^{2}$, because the unstable $\gamma$ phase disappeared during nitridation. In addition, the crater depth for the nitrided die steel was larger at a higher fluence of $F=0.12 \mathrm{~J} / \mathrm{cm}^{2} \mathrm{com}$ pared with that for the heat-treated die steel.

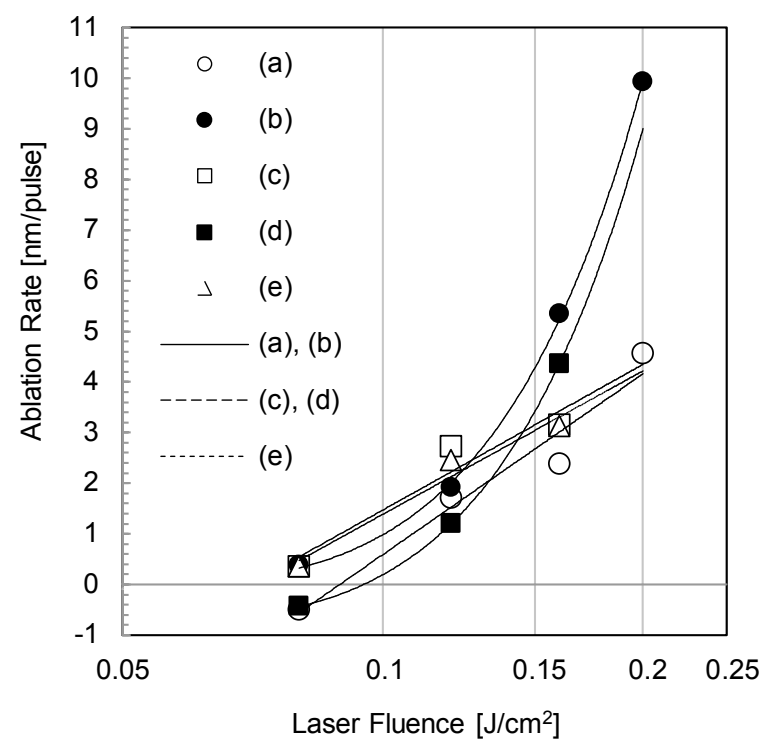

Fig. 7 Ablation rate obtained from the profiles of craters formed on (a) heat-treated die steel, (b) nitrided die steel, (c) untreated stainless steel, (d) nitrided stainless steel and (e) low-carbon steel, measured as a function of the laser fluence.
The ablation rate $L$, was calculated by dividing the crater depth by the number of laser shots. Figure 7 shows the ablation rates for the various steels plotted as a function of $F$ on a logarithmic scale. The ablation rate $L$ for the untreated stainless steel and low-carbon steel increased linearly from 0.34 to $3.1 \mathrm{~nm} /$ pulse with an increase in $F$, which is consistent with the theoretical formula [15]:

$$
L=1 / \alpha \ln \left(F / F_{t h}\right) \text {, }
$$

where $\alpha$ is the optical absorption coefficient and $F_{t h}$ is the threshold fluence for ablation.

The ablation rate for the heat-treated die steel observed at a low fluence of $F=0.08 \mathrm{~J} / \mathrm{cm}^{2}$ may be set to -0.5 $\mathrm{nm} /$ pulse by considering the height of the swelling as a negative depth. Therefore, it is considered that the ablation rate for the heat-treated die steel also increases linearly from -0.5 to $4.5 \mathrm{~nm} /$ pulse with an increase in $F$ from 0.08 to $0.2 \mathrm{~J} / \mathrm{cm}^{2}$.

In contrast, the ablation rate for the nitrided die steel was $0.36 \mathrm{~nm} /$ pulse, which is similar to those for the untreated stainless steel and low-carbon steel at a low fluence of $F=0.08 \mathrm{~J} / \mathrm{cm}^{2}$, was larger at a higher fluence of $F>$ $0.16 \mathrm{~J} / \mathrm{cm}^{2}$. Furthermore, it became clear that the dependence of the ablation rate on the fluence for the nitrided die steel increased as a second order curve, as shown in Fig. 7 (b). Its curve can be expressed as:

$$
L=1 / \alpha\left[\ln \left(F / F_{t h}\right)\right]^{2} \text {, }
$$

Figure 7(d) shows that the ablation rate for the nitrided stainless steel is negative at $-0.5 \mathrm{~nm} /$ pulse with a low fluence of $F=0.08 \mathrm{~J} / \mathrm{cm}^{2}$ because of the swelling, as with the heat-treated die steel. It should be noted that the dependence of the ablation rate on the fluence for the nitrided stainless steel also increased as a second order curve (Fig. 7(d)). Therefore, the high ablation rate observed at large $F$ and the second order curve fit may be considered characteristic of fs-laser ablation of a nitrided steel surface.

Hashida et al. reported that the dependence of the ablation rate on the fluence for copper is expressed by Eq. (1) in the range of low fluence under $0.22 \mathrm{~J} / \mathrm{cm}^{2}$, and is expressed by Eq. (2) in that of higher fluence beyond its value [16]. Though the range of the fluence in our paper is equivalent to that of low fluence, it may be considered that the dependence of the ablation rate on the fluence measured on the nitrided specimen surface comes to follow the Eq. (2).

\section{Conclusions}

The fs-laser-induced nanostructuring and ablation phenomena observed on nitrided alloy steels containing $\mathrm{Cr}$ was investigated. Heat-treated and plasma nitrided die steel $(1.5 \mathrm{C}-12 \mathrm{Cr})$ was mainly examined and nitrided stainless steel $(18 \mathrm{Cr}-8 \mathrm{Ni})$ and low-carbon steel $(0.1 \mathrm{C})$ were investigated for comparison. The minimum $D$ for $\mathrm{Cr}$ alloy steels observed at $F$ around the ablation threshold was $\sim \lambda / 3$. In contrast, $D$ for the low-carbon steel was larger. These results suggest that chromium nitride, carbide and oxide generated on the specimen surface induce a reduction in $D$. The ablation rates for nitrided alloy steels plotted against $F$ on a logarithmic scale were higher at larger $F$ and increased with a second order curve fit. 


\section{Acknowledgement}

A part of this work was supported by JSPS KAKENHI Grant-in-Aid for Scientific Research (B) Grant Number 24360312.

\section{References}

[1] M. Hudis: J. Appl. Phys., 44, (1973) 1489.

[2] N. Yasumaru: Materials Trans., Jap. Institute of Metals, 39, (1998) 1046.

[3] N. Yasumaru, K. Miyazaki and J. Kiuchi: Appl. Surf. Sci., 254, (2008) 2364.

[4] G. Miyaji and K. Miyazaki: Optics Express, 16, (2008) 16265.

[5] G. Miyaji, K. Miyazaki, K. Zhang, T. Yoshifuji and J. Fujita: Optics Express, 20, (2012) 14848.

[6] N. Yasumaru, E. Sentoku, K. Miyazaki, G. Miyaji and J. Kiuchi: Proc. 13th Int. Symp. on Laser Precision Microfabrication, Washington DC, (2012).

[7] N. Yasumaru, E. Sentoku, K. Miyazaki and J. Kiuchi: Appl. Surf. Sci., 264, (2013) 611.

[8] A. Y. Vorobyev, V. S. Makin and C. Guo: J. Appl. Phys., 101, (2007) 034903.
[9] A. Y. Vorobyev and C. Guo: J. Appl. Phys., 104, (2008) 063523.

[10]E. V. Golosov, A. A. Ionin, Yu. R. Kolobov, S. I. Kudryashov, A. E. Ligacher, Yu. N. Novoselov, L. V. Seleznev and D. V. Sinitsyn: J. Experimental \& Theoretical Phys., 113, (2011) 14.

[11]B. Huis in 't Veld, M. Groenendijk and H. Fischer: J. Laser Micro/Nanoengineering, 3, (2008) 206.

[12]L. Qi, K. Nishii and Y. Namba: Opt. Lett., 34, (2009) 1846.

[13]S. Hou, Y. Huo, P. Xiong, Y. Zhang, S. Zhang, T. Jia, Z. Sun, J. Qiu and Z. Xu: J. Phys. D, 44, (2011) 505401.

[14] N. Yasumaru, K. Miyazaki and J. Kiuchi: Appl. Phys. A, 76, (2003) 983; ibid, 81, (2005) 933.

[15]B.N. Chichkov, C. Momma, S. Nolte, F. von Alvensleben and A. Tunnermann: Appl. Phys. A, 63, (1996) 109.

[16] M. Hashida, A.F. Semerok, O. Gobert, G. Petite, Y. Izawa, J.F-. Wagner: Appl. Surf. Sci., 197-198, (2002) 862.

(Received: June 14, 2014, Accepted: December 9, 2014) 